\title{
Assuring long-term safety of highly effective gene- modulating therapeutics for rare diseases
}

\author{
Thomas 0. Crawford ${ }^{1,2}$ and Charlotte J. Sumner ${ }^{1,3}$ \\ Department of Neurology, ${ }^{2}$ Department of Pediatrics, and ${ }^{3}$ Department of Neuroscience, Johns Hopkins University School of Medicine, Baltimore Maryland, USA.
}

\begin{abstract}
A recent demonstration by Van Alstyne and colleagues (1) of long-term toxicity following short-term successful treatment of an animal model of spinal muscular atrophy (SMA) by gene transfer-mediated overexpression of survival motor neuron $(\mathrm{SMN})$ protein raises immediate questions for SMA patients, their families, and advising clinicians. This finding, however, also highlights the limits of safety data associated with any new therapy validated in small clinical trials of a rare disease as well as the potential inadequacy of current procedures for assuring long-term safety in gene-therapy programs under development. Optimal assessment of the safety of rare disease therapies may require improvements at multiple levels of the drug development, regulatory review, and postapproval monitoring processes.
\end{abstract}

\section{Short versus long-term toxicity}

A dramatic success for gene therapy of neurological disease occurred in 2019 when onasemnogene abeparvovec (OA) was found to be both safe and highly effective in treating infants with spinal muscular atrophy (SMA) (2), a recessively inherited motor neuron disease caused by loss-of-function mutations of the survival motor neuron 1 (SMN1) gene. OA consists of a constitutively active cytomegalovirus enhancer/chicken $\beta$ actin promoter driving a human SMN cDNA transgene packaged within a small nonpathogenic adenoassociated virus 9 (AAV9) vector. AAV9 has particular tropism for motor neurons following a single intravenous administration (3), and the transgene remains episomal, theoretically expressing indefinitely in postmitotic cells. Treated SMA infants, who otherwise would have been destined to relentless progressive weakness and early mortality, instead steadily improve. A principle focus in the development of $\mathrm{OA}$ and other gene-therapy programs has been on minimizing short-latency adverse events, including inflammatory and individual idiosyncratic reactions that likely relate to host immune responses to capsid proteins, transgene product, or both (4).

Although the possibility of viral host DNA integration and oncogenesis is a recognized long-term safety concern of gene therapy, Van Alstyne (1) raises a new potential "on-target" mechanism for the longterm toxicity of SMN gene therapy. They demonstrate that while SMA model mice injected with AAV9-SMN via i.c.v. delivery showed the expected amelioration of the motor neuron degeneration disease phenotype during the first weeks and months of disease, they also observed unexpected and novel deterioration in motor behavior between 6 and 10 months in a dosedependent manner. This was associated with loss of proprioceptive dorsal root ganglion (DRG) neurons and less severe loss of motor neurons. While overexpression of SMN protein has generally been considered to be benign (5), particularly as its stability is associated with incorporation into the multiprotein SMN complex (6), the Van Alsytyne report shows that the
Conflict of interest: TOC has been a consultant to Avexis/Novartis, Biogen, Cytokinetics, Cenentech, lonis, and Scholar Rock. He is/has been a site principal or co-principal investigator for the Biogen EMBRACE, NURTURE, and DEVOTE clinical trials, the Avexis/Novartis STR1VE and STRONG clinical trials, and individual clinical trials with Catabasis, Catalyst, Cytokinetics, Santherra, and Sarepta. CJS has been a consultant to Avexis, Novartis, lonis Pharmaceuticals, Biogen, PTC Therapeutics, Roche, Genentech, Cytokinetics, Sarepta, Nura Bio, and Atalanta. CJS received a research grant from lonis Pharmaceuticals in $2016(\$ 75,000)$ and currently receives grant support from Roche. CJS is a coholder of 2 pending patent applications (BIOLO274USA and BIOL0293WO) with lonis Pharmaceuticals on antisense oligonucleotides targeting SMN-AS1. CJS receives royalties from Elsevier for the book Spinal Muscular Atrophy: Disease Mechanisms and Therapy (editors, CJ Sumner, S Paushkin, CP Ko; Elsevier, 2017).

Copyright: @ 2021, American Society for Clinical Investigation.

Reference information: / Clin Invest. 2021;131(15):e152817. https://doi.org/10.1172/JCI152817. gene transfer-mediated high-expression levels of SMN protein in DRG and motor neurons cause SMN aggregation in the cytoplasm with sequestering of components of small nuclear ribonucleoproteins and altered splicing and gene-expression patterns. Together, these findings indicate that sufficiently high unregulated, longterm overexpression of SMN can itself cause neuronal degeneration.

This demonstration in an animal model of long-term toxicity following short-term successful therapy and the proposed mechanism raises plausible concern for similar long-term complications in OA-treated SMA patients. There are multiple reasons why this may not occur, including differences in the strengths of the different promoters used, higher levels of CNS transduction in the mouse likely with i.c.v. delivery, variations in capsid preparation methods, and the vast differences between mice and humans. Yet both OA and the mouse AAV9-SMA vector were designed to express high levels of SMN transcript using a constitutively active, artificial promoter with SMN cDNA lacking intronic or other regulatory elements. Lifetime high expression of SMN certainly contrasts with the developmental pattern of SMN expression in humans, which is high during fetal stages and declines and stabilizes following the early postnatal interval (7). Thus, while the risk for longterm toxicity from SMN overexpression in SMA patients treated with OA is likely low (8), the probability is unknown and the possibility impossible to exclude.

\section{How should families be counseled about the choices of therapy?}

In the last decade, SMA has been the fortunate recipient of an exceptional run of therapeutic successes. Patients and families now have a choice between two or three very different SMN protein-upregulating therapies, each of which have 
similar efficacies. The two other available SMA treatments act by modifying splicing of pre-mRNAs arising from the paralogous SMN2 gene. Nusinersen (9), FDA approved in 2016, is an antisense oligonucleotide administered by lumbar intrathecal injection. Risdiplam (10) is a small molecule splice modifier administered daily by mouth that was approved in 2020, but is not yet not approved for use in newborns. As all three treatments are most effective when started early, newborn screening for SMA is rapidly expanding across the United States and other countries.

Presently, the choice of therapy for SMA newborns is between OA and nusinersen. Prior to the Van Alstyne report, the specific cautions about OA focused on the known short-term complications. These include largely asymptomatic and manageable transient hepatic toxicity and the recent identification, in postmarketing surveillance, of thrombotic microangiopathy as a rare, severe hematologic complication (11). The vast majority of OA-treated infants do very well in the initial years following the therapy. The alternative therapy, nusinersen, has a longer and well established record of efficacy and safety, but has to be administered by ongoing, invasive intrathecal infusions. The choice of therapy thus generally focuses upon the perception of burden associated with the potential complications of each. OA presents as a putative "one-and-done" intravenous therapy, but with concern for known short-term mild toxicities, a known rare short-term severe idiosyncratic toxicity, and the new added concern of an unlikely but irreversible and difficult-to-define delayed toxicity. The alternative, nusinersen, has a record of safety, but is accompanied by the burden of lifetime thrice-yearly spinal taps.

Modern medical practice often requires choice in a setting of inadequate information. For families of newborns found by newborn screening to have SMA, this choice is inevitably complicated by a number of factors, including the initial shock of an unexpected genetic diagnosis, the need to understand highly complex biologic processes, the prospect of invasive medical interventions by specialists unfamiliar to the family in unfamiliar tertiary medical centers, the stress of obtaining authorization for very expensive therapy for a newborn who is not yet enrolled into an insurance plan, and all of this upon a background of urgency for action. To that now is added assumption of responsibility for a "sword of Damocles" delayed toxicity paradigm, where concern about a low-probability risk for a new form of neurodegeneration, given its unknowable latency, can never be fully resolved. Clinicians need be well versed in the known burden and benefits of each therapy, and updatable and accessible educational materials for families that outline these choices are needed. In addition, standardof-care parameters for this counseling need to be established for SMA therapeutics as well as other emerging genetic treatments. Clinicians also need be mindful that during this difficult time, the choice itself is stressful and raises concern about a perpetual burden of responsibility for any ill consequences that might arise from the choice made. Additional support from patient-service organizations and from medical, scientific, and pharmaceutical communities will be invaluable.

\section{What steps should be taken to monitor for long-term toxicity of SMA and other gene therapies?}

Surveillance for long-term neurologic toxicity is often difficult because of inherent functional accommodations that conceal early pathology. In the young, toxic effects can be further concealed by the shifting baseline of normal development. In those with the infantile form of SMA, the onset of neurodegeneration appears to be before birth (12), genetic diagnosis, or the appearance of overt symptoms and thus before the initiation of SMN-enhancing therapy. Given this early pathology, distinguishing on clinical grounds alone those early acquired deficits of SMA that are unveiled by the passage of time and normal development (13) from the motor deficits that increase because of increasing pathology of late-developing toxicity may be virtually impossible. An additional challenge to recognizing late toxicity is the potential of high subject-to-subject variability. Differences in maturation of the blood-brain barrier may substantially alter CNS exposure to the transgene: the initial treated 50 subjects in the two enabling clinical trials
$(2,14)$ mostly received treatment later than those who receive OA following diagnosis by newborn screening.

Given the inability to withdraw gene therapy once administered and the impossibility of exhaustively vetting for delayed toxicity during preclinical development, there is an ethical imperative to commit resources to a continuance of investigations well after regulatory approval. These long-term toxicity investigations will be as challenging as present efforts to minimize short-term complications. The difficult process of securing long-term safety for gene-modulating and other specific therapies for rare diseases will require commitments from all stakeholders. Optimally, it involves creation of at least the following specific resources and programs:

(a) Long-term animal safety research. Pharmaceutical companies need to initiate and maintain long-term safety experiments in a range of species, including nonhuman primates. These experiments should be continued after approval, continually updated, and accessible to the public as demonstration of continuing good faith.

(b) Prospective mandatory surveillance for a broad range of toxicity. Voluntary reporting and optional participation registries are at best insensitive tools for identifying long-term complications (15). The FDA, through the Risk Evaluation and Mitigations Strategy program (16), requires pharmaceutical companies to develop and commit to a negotiated risk-mitigation strategy for therapies associated with a known severe complication; mitigation plans can include extended surveillance. Absent a known complication, however, the FDA lacks regulatory authority to compel a mandatory registry having both pharmaceutical and public oversight.

(c) A biorepository of samples. Multiple stakeholders should participate and support creation and curation of a broad sample collection to enable further therapy-specific and disease-specific biomarker development.

(d) A rapid standardized autopsy program. In the case of SMA, we can unfortunately expect there will continue to be deaths of individuals receiving SMN-specific therapies because many received these treatments late in the course of their neurodegeneration. Essential to success is 
rapid deployment of appropriate personnel and materials on short notice to broad geographic regions, as optimal acquisition and management of collected tissues goes well beyond conventional autopsy procedures (7). Further, success of such a program depends upon awareness and support of caring medical providers, whose commitment to the effort in turn can best be assured by the support they receive from pharma, neuromuscular specialty consultants, and patient-advocacy organizations.

(e) Investigations of strategies to counter potential mechanisms of long-term toxicity. (As an example, see ref. 17.)

\section{How are these concerns of larger importance?}

The experience of serious long-term toxicity following widespread popular acceptance of a therapy has been an unfortunate theme of the history of medicine. The experience has sculpted the process of both therapeutics development and therapeutics regulation. Many of the most dramatic examples, such as the teratogenicity of thalidomide following treatment of hyperemesis gravidarum (18), the cervical dysplasia following diethylstilbestrol treatment for prevention of miscarriage (19), or epidemic thyroid cancer following thymic irradiation intended to reduce risk for sudden infant death (20), not only highlight the importance of long-term monitoring of novel therapeutics, but also the special biologic vulnerabilities of development and the extended time necessary for development to unveil toxic consequences.

The recent remarkable success of $\mathrm{OA}$ as a treatment of SMA heralds an explosion of similar gene-therapy programs targeting virtually the whole spectrum of rare genetic disease. The success, in a very short interval, of now three therapies for this one disease also reveals the inadequacies of the traditional large-market therapeutics development and regulatory processes processes that have served reasonably well, but evolved before the present emergence of personalized medicines. The present system of rare disease therapeutics development, regulatory review, and postapproval surveillance leaves a void of information about long-term safety that patients and their families need to make a choice. There is need for a broad commitment to improving therapeutic development for rare diseases, to reduce both the probability of late complications and the delay in recognition of such complications.

\section{Acknowledgments}

The authors express their appreciation to Richard Finkel and Julie Parsons for critical review and suggestions.

Address correspondence to: Thomas O. Crawford, 200 North Wolfe Street, Rubenstein Child Health Building 2145, Baltimore, Maryland 21287, USA. Phone: 410.955.4259; Email: tcrawfo@jhmi.edu.

1. Van Alstyne M, et al. Gain of toxic function by long-term AAV9-mediated SMN overexpression in the sensorimotor circuit [published online April 1, 2021]. Nat Neurosci. https://doi. org/10.1038/s41593.021.00827-3.

2. Mendell JR, et al. Single-dose gene-replacement therapy for spinal muscular atrophy. $\mathrm{N} \mathrm{Engl} \mathrm{J}$ Med. 2017;377(18):1713-1722.

3. Foust KD, et al. Rescue of the spinal muscular atrophy phenotype in a mouse model by early postnatal delivery of SMN. Nat Biotechnol. 2010;28(3):271-274.

4. Nidetz NF, et al. Adeno-associated viral vectormediated immune responses: understanding barriers to gene delivery. Pharmacol Ther. 2020;207:107453.

5. Gavrilina TO, et al. Neuronal SMN expression corrects spinal muscular atrophy in severe SMA mice while muscle-specific SMN expression has no phenotypic effect. Hum Mol Genet. 2008;17(8):1063-1075.

6. Burnett BG, et al. Regulation of SMN protein stability. Mol Cell Biol. 2009;29(5):1107-1115.

7. Ramos DM, et al. Age-dependent SMN expression in disease-relevant tissue and implications for SMA treatment. J Clin Invest. 2019;129(11):4817-4831

8. Finkel RS, Fischbeck KH. Maybe too much of a good thing in gene therapy [published online June 15, 2021]. Nat Neurosci. https://doi. org/10.1038/s41593.021.00882-w.

9. Finkel RS. Nusinersen versus sham control in infantile-onset spinal muscular atrophy. $N$ Engl J Med. 2017;377(18):1723-1732.

10. Baranello G. Risdiplam in type 1 spinal muscular atrophy. N Engl J Med. 2021;384(10):915-923.

11. Chand DH, et al. Thrombotic microangiopathy following onasemnogene abeparvovec for spinal muscular atrophy: a case series. JPediatr. 2021;231:265-268.

12. Kong L, et al. Impaired prenatal motor axon development necessitates early therapeutic intervention in severe SMA. Sci Transl Med. 2021;13(578):eabb6871.

13. De Vivo DC, et al. Nusinersen initiated in infants during the presymptomatic stage of spinal muscular atrophy: interim efficacy and safety results from the phase 2 NURTURE study. Neuromuscul Disord. 2019;29(11):842-856.

14. Day JW, et al. Onasemnogene abeparvovec gene therapy for symptomatic infantile-onset spinal muscular atrophy in patients with two copies of SMN2 (STR1VE): an open-label, single-arm, multicentre, phase 3 trial. Lancet Neurol. 2021;20(4):284-293.

15. Fontanarosa PB, et al. Postmarketing surveillance--lack of vigilance, lack of trust. JAMA. 2004;292(21):2647-2650.

16. Guadamuz JS, et al. Use of risk evaluation and mitigation strategies by the US Food and Drug Administration, 2008-2019. JAMA. 2020;324(3):299-301.

17. Hordeaux J, et al. MicroRNA-mediated inhibition of transgene expression reduces dorsal root ganglion toxicity by AAV vectors in primates. $\mathrm{Sci}$ Transl Med. 2020;12(569):eaba9188.

18. Vargesson N. Thalidomide-induced teratogenesis: history and mechanisms. Birth Defects Res C Embryo Today. 2015;105(2):140-156.

19. Goodman A, et al. The long-term effects of in utero exposures--the DES story. $\mathrm{NEngl} \mathrm{J} \mathrm{Med.}$ 2011;364(22):2083-2084.

20. Adams MJ, et al. Thyroid cancer risk $40+$ years after irradiation for an enlarged thymus: an update of the Hempelmann cohort. Radiat Res. 2010;174(6):753-762. 